\title{
Eigen-Vector Based Leads for Reconstruction of the 12-Lead Electrocardiogram
}

\author{
DD Finlay ${ }^{1}$, CD Nugent ${ }^{1}$, MP Donnelly ${ }^{1}$, SP Nelwan ${ }^{2}$ \\ ${ }^{1}$ Univeristy of Ulster, Belfast, Northern Ireland, UK \\ ${ }^{2}$ Erasmus Medical Centre, Rotterdam, Netherlands
}

\begin{abstract}
In this study we have developed a limited lead system to allow reconstruction of the 12-lead ECG. This has been based upon the analysis of eigen-vectors calculated from body surface potential map (BSPM) data. Eigenvectors were calculated from a set of 117 lead BSPMs (normal $=172, L V H=178, M I=209)$. The extrema of the first three eigen-vectors were used to determine the positions of recording sites for three bipolar leads. These three bipolar leads were then used to reconstruct 12-lead ECGs recorded from an unseen group of subjects (normal $=57, L V H=59, M I=69$ ). It was found that the suggested leads could reconstruct the 12-lead ECG with a median RMS error of $57.0 \mu \mathrm{V}$ and a correlation coefficient of 0.968. This was in comparison to the EASI leads, which, when tested on the same data, produced a median RMS error of $60.1 \mu \mathrm{V}$ and a correlation coefficient of 0.955. Following comparison on a lead-bylead basis the eigen-vector based leads were found to perform particularly well in the reconstruction of the precordial leads V1-V5. Further work is required to refine and to determine the practical utility of the proposed leads in practice.
\end{abstract}

\section{Introduction}

In electrocardiography limited or derived lead systems are currently stimulating much interest. These systems offer the potential to record the ECG from fewer sites than would normally be used. Information from missing sites is then estimated using transformation coefficients [1].

Several systems have been reported in the literature which allow the reconstruction of the 12-lead ECG from a reduced number of recording sites. One example is the EASI system [2,3], where the 12-lead ECG is derived from three bipolar leads based on the Frank VCG system [4]. Several systems have also been reported that use a subset of the 12-lead ECG [5-7]. These systems use information from the limb leads along with just two precordial leads to derive the four missing precordial leads. Despite the commercial availability of these systems and the clinical interest there is still some controversy and reluctance surrounding their widespread use.

In this study we develop a limited lead system to allow reconstruction of the 12-lead ECG based on the analysis of eigen-vectors which have been calculated from body surface potential maps (BSPMs). Eigen-vector based techniques have been previously used in the analysis of BSPMs. This has included the compression of BSPMs [8], and selection of unipolar leads for limited lead BSPMs [9]. In this study we employ eigen-vector based techniques to find statistically orthogonal bi-polar leads.

\section{Methods}

We calculated eigen-vectors for a set of 117 lead BSPMs. The recording apparatus and procedure has previously been described in [10-11]. Briefly, the electrode array used to record the BSPMs comprised of 117 recording sites distributed over the surface of the thorax as shown in Figure 1. These recording sites were sampled simultaneously with respect to the Wilson Central Terminal derived from three standard (distal) limb leads.



Figure 1. Electrode configuration used to record 117 lead BSPMs. Also depicted are the locations of the six precordial leads relative to the 117 lead array.

The dataset used consisted of 744 recordings taken from normal subjects, subjects who had suffered myocardial infarction (MI) and subjects with left ventricular hypertrophy $(\mathrm{LVH})$. For each subject 
averaged beats were calculated for each lead. Prior to analysis the dataset was partitioned to facilitate experimentation and evaluation. The experimentation dataset consisted of data from 559 subjects (normal = $172, \mathrm{LVH}=178, \mathrm{MI}=209$ ). The evaluation dataset consisted of data from the remaining 185 subjects (normal $=57, \mathrm{LVH}=59, \mathrm{MI}=69$ ).

Eigen-vectors were calculated from all map frames (PQRST) for all subjects in the experimental dataset. The resulting eigen-vectors exist in the same space as the 117 lead BSPM frames and therefore can be plotted as contour maps. The first six eigen-vectors, ranked based on eigen-value are plotted in Figure 2. The maps exhibit a similar pattern as that reported by previous investigators [7]. Namely, the first three eigen-vectors exhibit a dipolar pattern whereas subsequent eigen-vectors exhibit a more complex pattern with more than two poles.

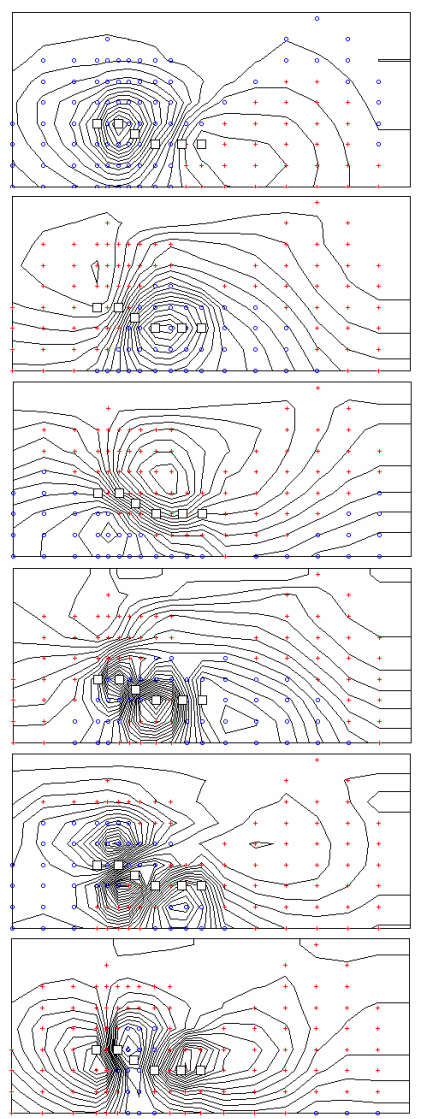

Figure 2. Top six eigen-vectors (descending order from top) plotted as contour maps.

Bipolar leads were determined based on the positive and negative pole of each eigen-vector. The bipolar leads resulting from the first three eigen-vectors are illustrated in Figure 3.

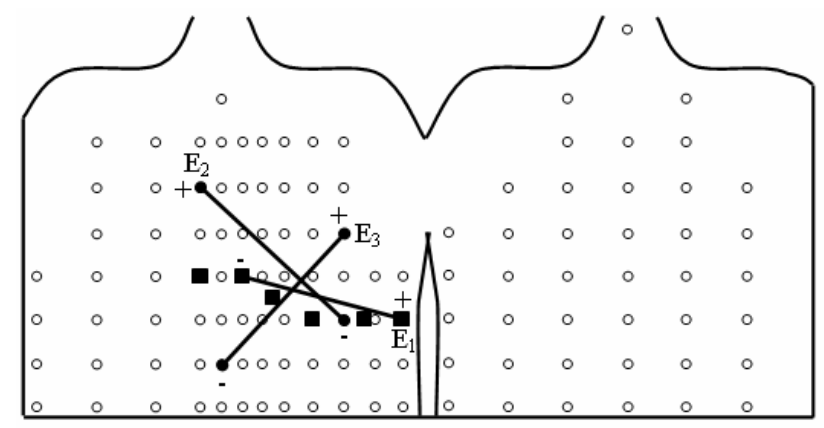

Figure 3. Three bipolar leads as determined by locations of extrema on eigen-vectors shown in Figure 2.

A set of transformation coefficients were derived that allowed each lead of the 12-lead ECG to be derived from the three eigen-leads. These were derived by performing a linear regression, again using the experimentation dataset. The 12-lead ECG was then derived from the three eigen-leads for each subject in the evaluation dataset. These derived 12-lead ECGs were compared with the actual 12-lead ECGs for that population. Two independent and widely used metrics were used for comparative purposes. RMS error, as described in equation 1, was calculated to provide a measure of the average potential error between the two ECG waveforms.

$$
R M S E=\sqrt{\frac{\sum_{i=1}^{n}\left(x_{1, i}-x_{2, i}\right)^{2}}{n}}
$$

where $x_{1, i}$ and $x_{2, i}$ are the $i$ th sample from a measured and estimated ECG lead respectively and $n$ is the number of samples.

Correlation coefficient, as described in equation 2, was used to provide a measure of the similarity in the shape of the measured versus estimated ECG waveforms.

$$
\rho=\frac{P_{1} P_{2}}{\left|P_{1}\right|\left|P_{2}\right|}
$$

where $P_{1}$ and $P_{2}$ are the measured and estimated ECG leads respectively. Both measures were determined for each lead of each patient. The median and interquartile ranges were then calculated for each lead across all patients. For the purposes of comparison the performance of the EASI [2] leads and the Nelwan [5] lead system were also calculated for the evaluation dataset.

The Wilcoxon's signed rank test was used as a measure of statistical significance of the difference in evaluating the performance between different lead sets. This test was applied to the paired difference in performance for the various experiments for each subject in the evaluation dataset. 


\section{Results}

The reconstruction accuracy of the eigen-leads is shown in Figure 4. This set of error bars shows the median and interquartile range for each reconstructed lead. The performance of the EASI and Nelwan lead systems has also been included. Figures $4 \mathrm{a}$ and $4 \mathrm{~b}$ illustrate RMS error and correlation coefficient respectively.

The median RMS error across all reconstructed leads for the Eigen based, EASI and Nelwan lead systems was 57.0 $\mu \mathrm{V}, 60.1 \mu \mathrm{V}$ and $72.5 \mu \mathrm{V}$ respectively. The respective median correlation coefficient across all reconstructed leads was 0.968, 0.955 and 0.981, respectively.

It can be seen in Figure 4a that the eigen-leads exhibit a consistent level of reconstruction accuracy across all reconstructed leads with a deviation in median of less than $30 \mu \mathrm{V}$. In contrast, the error observed in the reconstruction of the 12-lead ECG using the EASI leads is seen to fluctuate by almost $150 \mu \mathrm{V}$. This is evident with a visible increase in error when reconstructing the precordial leads $V_{1}$ to $V_{5}$ and especially leads $V_{2}-V_{4}$. The Nelwan lead system which does not reconstruct any limb leads shows a deviation of approximately $40 \mu \mathrm{V}$ across the four reconstructed leads.

Following the Wilcoxon's test it was found that the eigen-leads significantly out performed the EASI leads in

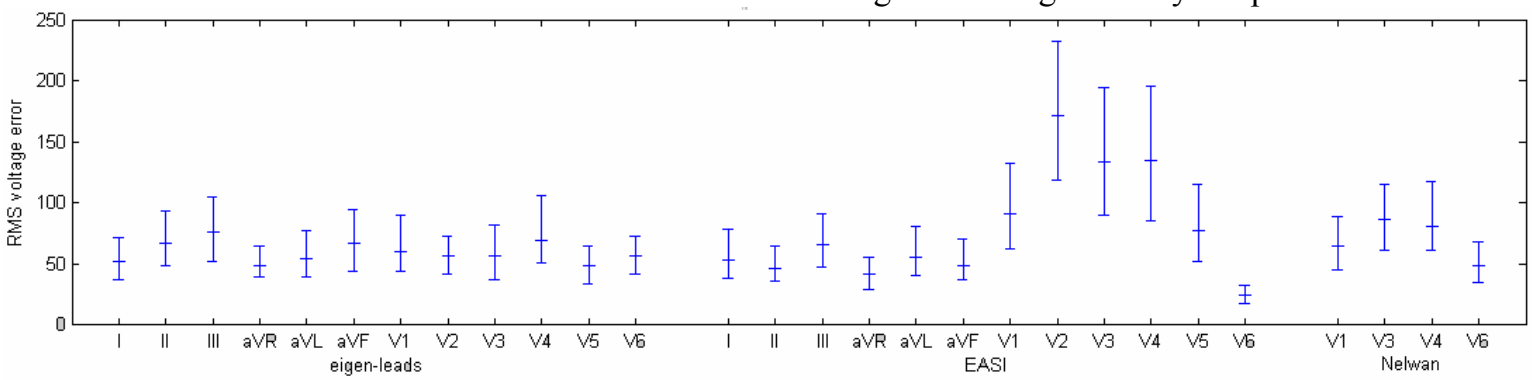

(a)

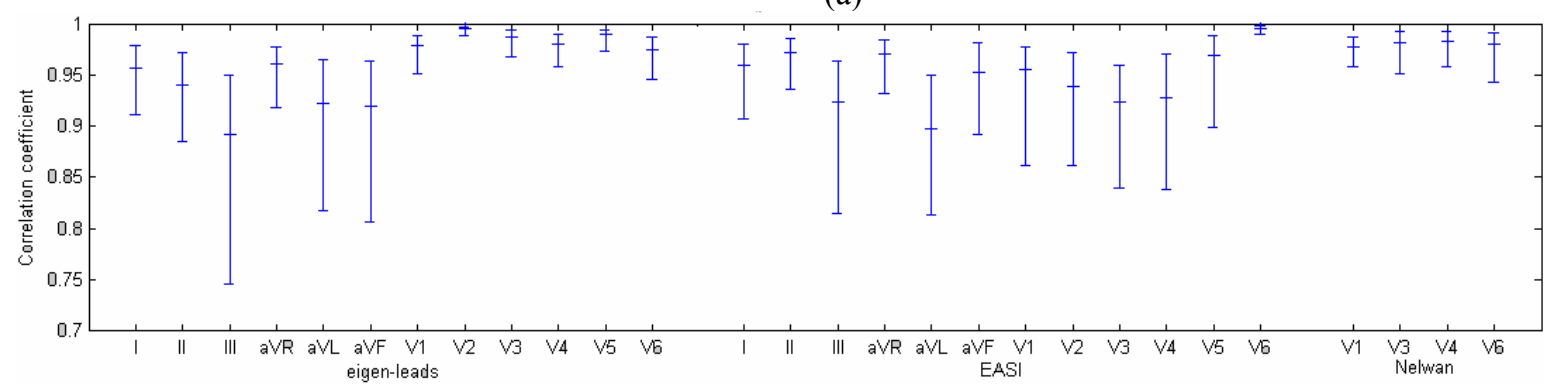

(b)

Figure 4. Performance of eigen-leads, EASI leads and Nelwan leads when reconstructing the 12-lead ECG. Reconstruction accuracy for each reconstructed lead is shown (median and interquartile range). Figure 4a indicates RMS error, Figure $4 \mathrm{~b}$ indicates correlation coefficient.

the reconstruction of the precordial leads $\mathrm{V}_{1}$ through $\mathrm{V}_{5}(p<0.001)$. There was, however, a significant deficit in the reconstruction accuracy of lead $\mathrm{V}_{6}$ using the eigenleads as compared to the EASI leads $(p<0.001)$. The EASI leads outperform the eigen-leads in the reconstruction of all the limb leads with a significant margin $(p<0.05)$. This is with the exception of lead aVL where the eigen-leads actually yield a more accurate reconstruction, however, this improvement was not found to be significant $(p=0.265)$. When compared to the Nelwan leads, the eigen-leads reconstructed $V_{1}$ with a similar margin of error $(p=0.166)$. Of the remaining leads, $V_{3}$ and $V_{4}$ were more accurately reconstructed using the eigen-leads and $\mathrm{V}_{6}$ was more accurately reconstructed using the Nelwan leads (in all three cases $p<0.001)$.

When the error bars for correlation coefficient, in Figure $4 b$, are examined, it can be seen that the eigenleads again fair favorably when reconstructing precordial leads. There was, however, more variability in the correlation coefficient values for precordial leads versus those for limb leads using the eigen-leads than was evident in the RMS voltage error. Such a deficit will be more evident when performance is expressed in terms of correlation coefficient given that the signal levels on limb leads are generally lower than that of precordial leads and although voltage differences will be smaller in these leads pattern difference may be more extreme.

When comparing the reconstruction accuracy of the eigen-leads versus the other two lead systems, based on 
correlation coefficient, , the eigen-leads generally outperform the EASI leads in the reconstruction of precordial leads $\mathrm{V}_{1}-\mathrm{V}_{5}(p<0.001)$. As was evident in the RMS voltage error lead $\mathrm{V}_{6}$ is more accurately reconstructed using the EASI leads $(p<0.001)$. A similar pattern as was exhibited in the RMS voltage errors is also observed here for the limb leads, as again, the majority of leads are equally as well if not more accurately reconstructed using the EASI leads. When compared to the Nelwan leads the eigen-leads reconstructed $\mathrm{V}_{1}$ and $\mathrm{V}_{4}$ with similar accuracy $(p=0.479$ and $p=0.138$ respectively). $\mathrm{V}_{3}$ was more accurately reconstructed using eigen-leads $(p<0.001)$ whilst $\mathrm{V}_{6}$ was more accurately reconstructed using the Nelwan leads $(p<0.001)$. This is similar to the pattern of performance observed based on the RMS voltage error.

\section{Discussion and conclusions}

Here we have illustrated the performance of a set of orthogonal leads for reconstructing the 12-lead ECG. These leads can be thought of as being statistically orthogonal as the eigen-vector based approach exploits this characteristic of the studied dataset (statistical orthogonality). This contrasts the EASI lead system which is based on spatial orthogonality.

The eigen-leads have been shown to perform reasonably well in the reconstruction of the 12-lead ECG particularly when compared to the EASI leads. This is most evident in the reconstruction of the precordial leads. This quality may make these leads suitable for applications where the precordial lead information is sought without the recording overhead of the Wilson Central Terminal leads.

There are still some issues with the proposed system that require further investigation. Firstly, the eigen-leads currently need six recording sites which is more than that of the other limited lead systems. A way of addressing this could be to combine poles of some of the eigen-leads to reduce the number of required recording sites. This may be to the detriment of reconstruction performance but may be desired for reasons of practicality. Secondly, the practical limitations of this approach are yet to be tested, specifically, we have not identified anatomical landmarks that allow for the accurate application of the leads. An issue that is related to this that also needs clarification is the fact that we have not yet assessed the effects of electrode placement variability on the reconstruction performance. All of these issues form the basis of the plans for our further work.

\section{Acknowledgements}

The authors would like to acknowledge the support of Professor Robert Lux who helped develop the study. We would also like to thank Dr Fred Kornreich who provided the BSPM data.

\section{References}

[1] Finlay DD, Nugent CD, Kellett JG, Donnelly MP, McCullagh PJ, Black ND. Synthesising the 12-lead electrocardiogram: Trends and challenges. Eur.J.Intern.Med. 2007 Dec;18(8):566-570.

[2] Dower GE, Yakush A, Nazzal SB, Jutzy RV, Ruiz CE. Deriving the 12-lead electrocardiogram from four (EASI) electrodes. J.Electrocardiol. 1988;21 Suppl:S182-7.

[3] Feild DQ, Feldman CL, Horacek BM. Improved EASI coefficients: their derivation, values, and performance. J.Electrocardiol. 2002;35 Suppl:23-33.

[4] Frank E. An accurate, clinically practical system for spatial vectorcardiography. Circulation 1956; 13: 737-49.

[5] Nelwan SP, Kors JA, Meij SH. Minimal lead sets for reconstruction of 12-lead electrocardiograms. J Electrocardiol 2000;33 Suppl:163-166.

[6] Drew BJ, Pelter MM, Brodnick DE, Yadav AV, Dempel D, Adams MG. Comparison of a new reduced lead set electrocardiogram with the standard electrocardiogram for diagnosing cardiac arrhythmias and myocardial ischemia. J Electrocardiol 2002;35 (Suppl):13-21.

[7] Wei D, Kojima T, Nakayama T, Sakai Y. US Patent No. 6,721,591: Method of deriving standard 12-lead electrocardiogram and electrocardiogram monitoring apparatus. US Patent Office April 2004;600/512.

[8] Lux RL, Evans AK, Burgess MJ, Wyatt RF, Abildskov JA. Redundancy reduction for improved display and analysis of body surface potential maps. I. Spatial compression. Circ.Res. 1981 Jul;49(1):186-196.

[9] Barr RC, Spach MS, Herman-Giddens GS. Selection of the number and positions of measuring locations for electrocardiography. IEEE Trans.Biomed.Eng. 1971 Mar;18(2):125-138.

[10] Kornreich F, Montague TJ, Rautaharju PM. Identification of first acute $Q$ wave and non-Q wave myocardial infarction by multivariate analysis of body surface potential maps. Circulation 1991 Dec;84(6):2442-2453.

[11] Montague TJ, Smith ER, Cameron DA, Rautaharju PM, Klassen GA, Felmington CS, et al. Isointegral analysis of body surface maps: surface distribution and temporal variability in normal subjects. Circulation 1981 May;63(5):1166-1172.

Address for correspondence

Dewar Finlay

University of Ulster

Shore Road

Belfast

BT37 0QB

E-mail address d.finlay@ulster.ac.uk 